\title{
Precision Measurements of Fundamental Interactions with (Anti)Neutrinos
}

\section{R. Petti*}

Department of Physics and Astronomy, University of South Carolina, Columbia SC 29208, USA

E-mail: roberto.petti@cern.ch

\begin{abstract}
We discuss the main limitations of past neutrino scattering experiments and possible ways to address them in a next-generation program of precision measurements of fundamental interactions with (anti)neutrinos. A reduction of the longstanding precision gap with respect to electron scattering experiments could provide interesting synergies with the existing efforts in the fixed-target, collider, and nuclear physics communities.
\end{abstract}

XXVII International Workshop on Deep-Inelastic Scattering and Related Subjects - DIS2019 8-12 April, 2019

Torino, Italy

${ }^{*}$ Speaker. 


\section{Limitations of Neutrino Scattering Experiments}

Neutrinos and antineutrinos have many desirable properties including being sensitive only to weak interactions, full polarization, and a complete flavor separation $(d / u, s / \bar{s}, \bar{d} / \bar{u}$, valence/sea) through the Charged Current (CC) process. These features makes them an ideal probe for electroweak physics and for the structure of nucleons and nuclei. However, this unique potential has been only partially explored due to four major limitations of $v(\bar{v})$ scattering experiments.

Statistics vs. resolution: The need of massive detectors to compensate for the tiny cross-sections implies that neutrino experiments are usually a compromise between the collected statistics (target mass) and the resolutions achievable in the reconstruction of the interactions. The table below shows a summary of the main features of some of the past and present neutrino experiments. The largest samples correspond to about $10^{7} v_{\mu} \mathrm{CC}$ events. Only a few experiments achieved uncertainties on the muon energy scale, $\Delta E_{\mu}$, at the sub-percent level, at the price of smaller statistics.

Targets: Most neutrino experiments are based upon massive detectors, consisting of several different materials and components, providing at the same time the active detection system and the neutrino target mass. A drawback of this configuration is the difficulty to precisely know the target chemical composition and mass, limiting the ultimate precision achievable in the measurements. Additional potential issues are the difficulty to modify the target configuration during data taking and/or the limited options for the target materials.

Fluxes: The energy of the incoming (anti)neutrino is unknown on an event-by-event basis and typically can vary over a broad range in conventional wide band neutrino beams. For this reason neutrino experiments have been affected by relatively large (5-10\%) systematic uncertainties on the knowledge of the (anti)neutrino fluxes.

Nuclear effects: Heavy nuclear targets are required to collect large statistics, but introduce additional systematic uncertainties with respect to elementary targets [1]. The unknown (anti)neutrino energy must be inferred from the detected final state particles originated in the interactions, which are affected by a substantial nuclear smearing. The corresponding unfolding process typically implies Monte Carlo and/or model corrections depending upon a number of different parameters, often empirically tuned with the observed kinematic distributions [2]. These corrections can be particularly critical in regions with small acceptance or large variations of the kinematics.

As a result, a major precision gap is present between (anti)neutrino experiments and the electron experiments performed at JLab, SLAC, HERA, LEP, etc. The four issues described above represent intrinsic limitations of current (anti)neutrino scattering experiments, making an accurate

\begin{tabular}{l|c|c|c|c|c|c} 
Experiment & Mass & $v_{\mu}$ CC Stat. & Target & $E_{v}(\mathrm{GeV})$ & $\Delta E_{\mu}$ & $\Delta E_{\mathrm{H}}$ \\
\hline \hline CDHS & $750 \mathrm{t}$ & $10^{7}$ & $\mathrm{p}, \mathrm{Fe}$ & $20-200$ & $2.0 \%$ & $2.5 \%$ \\
BEBC & various & $5.7 \times 10^{4}$ & $\mathrm{p}, \mathrm{D}, \mathrm{Ne}$ & $10-200$ & & \\
CCFR & $690 \mathrm{t}$ & $1.0 \times 10^{6}$ & $\mathrm{Fe}$ & $30-360$ & $1.0 \%$ & $1.0 \%$ \\
NuTeV & $690 \mathrm{t}$ & $1.3 \times 10^{6}$ & $\mathrm{Fe}$ & $30-360$ & $0.7 \%$ & $0.43 \%$ \\
CHORUS & $100 \mathrm{t}$ & $3.6 \times 10^{6}$ & $\mathrm{Emul} ., \mathrm{Pb}$ & $10-200$ & $2.5 \%$ & $5.0 \%$ \\
NOMAD & $2.7 \mathrm{t}$ & $1.5 \times 10^{6}$ & $\mathrm{C}, \mathrm{Fe}$ & $5-200$ & $0.2 \%$ & $0.5 \%$ \\
MINOS ND & $980 \mathrm{t}$ & $3.6 \times 10^{6}$ & $\mathrm{Fe}$ & $3-50$ & $2-4 \%$ & $5.6 \%$ \\
T2K ND & $1.9 \mathrm{t}$ & $10^{5}$ & $\mathrm{CH}, \mathrm{H}_{2} \mathrm{O}$ & $0.2-5$ & $0.6 \%$ & $2-4 \%$ \\
MINER $v \mathrm{~A}$ & $5.4 \mathrm{t}$ & $10^{7}$ & $\mathrm{CH}, \mathrm{C}, \mathrm{Fe}, \mathrm{Pb}$ & $1-30$ & $2 \%$ & \\
\hline
\end{tabular}




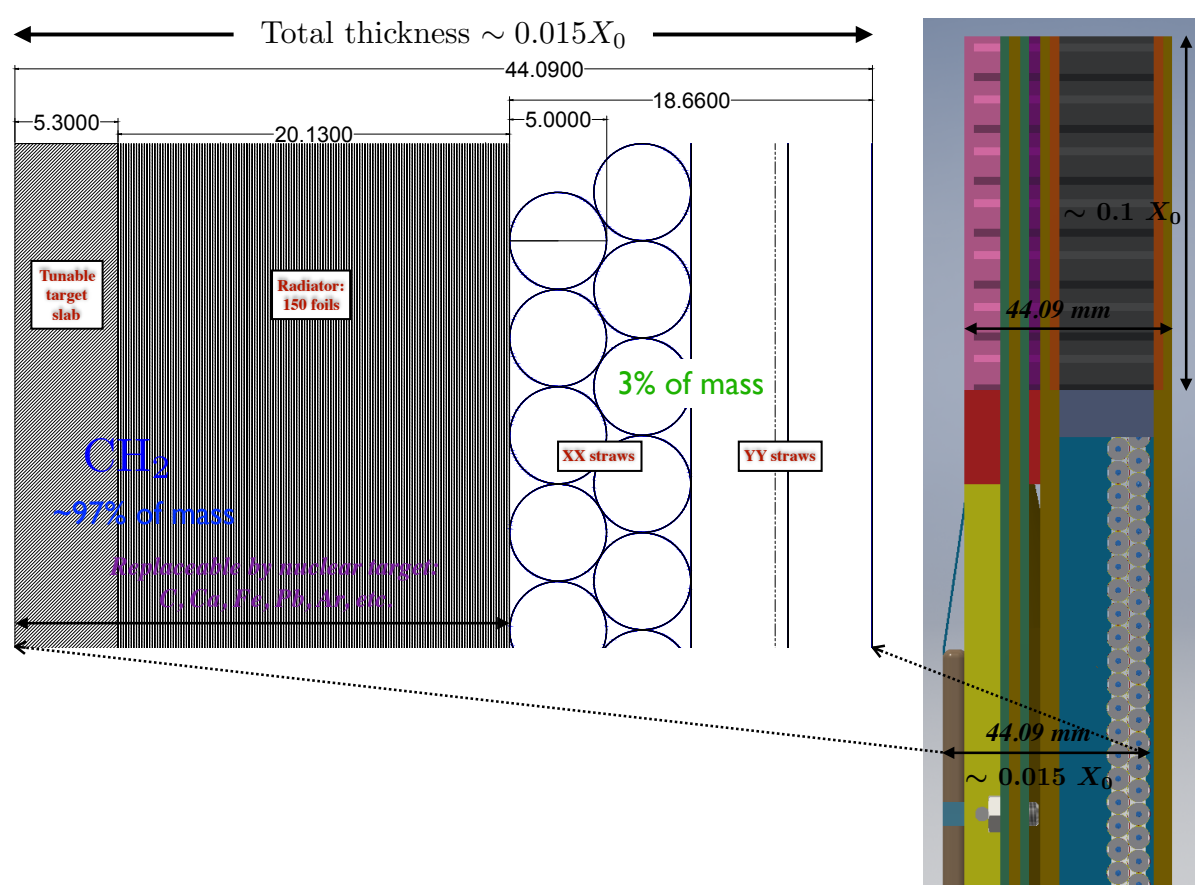

Figure 1: Drawing of a STT module allowing a control of the configuration, chemical composition, and mass of the $v(\bar{v})$ target(s) comparable to electron scattering experiments.

detection of (anti)neutrino interactions extremely challenging. This difficulty is illustrated by the many outstanding discrepancies among existing measurements performed by different experiments, as well as with various theoretical models [1]. Any sensible program of precision measurements of fundamental interactions with (anti)neutrinos must first address each of those outstanding issues.

\section{New Perspectives on Precision (Anti)Neutrino Scattering}

\subsection{Statistics and Resolution}

The Long-Baseline Neutrino Facility (LBNF) is expected to provide $v \& \bar{v}$ beams of unprecedented intensity, with a nominal beam power of $1.2 \mathrm{MW}$ and $1.1 \times 10^{21} \mathrm{pot} / \mathrm{year}$. Although primarily designed for the study of long-baseline neutrino oscillations, this facility offers a unique opportunity for neutrino scattering physics $[3,4]$, due to the availability of a high-energy beam option optimized to detect the $v_{\tau}$ appearance from neutrino oscillations, in addition to the default low-energy beam optimized for the search for $\mathrm{CP}$ violation. It is conceivable to have a dedicated 2 year run with this high energy beam after the completion of the nominal data taking of $5+5$ years with the default low-energy neutrino and antineutrino beams [3,4]. By that time the planned upgrades of the beam intensity to a nominal power of $2.4 \mathrm{MW}$ would more than double the available statistics. Under such conditions, a detector with a fiducial mass of about 5 tons would collect more than $10^{8} \mathrm{CC}$ interactions at the near site (Fig. 2). Given this relatively compact size, we can afford a high resolution detector providing an accurate measurement of the four-momenta of all final state particles produced in (anti)neutrino interactions and $\Delta E_{\mu} \leq 0.2 \%$.

\subsection{Control of Targets}

A detector technology designed to offer a control of the configuration, chemical composition and mass of the neutrino targets similar to the one achieved in electron scattering experiments is a 


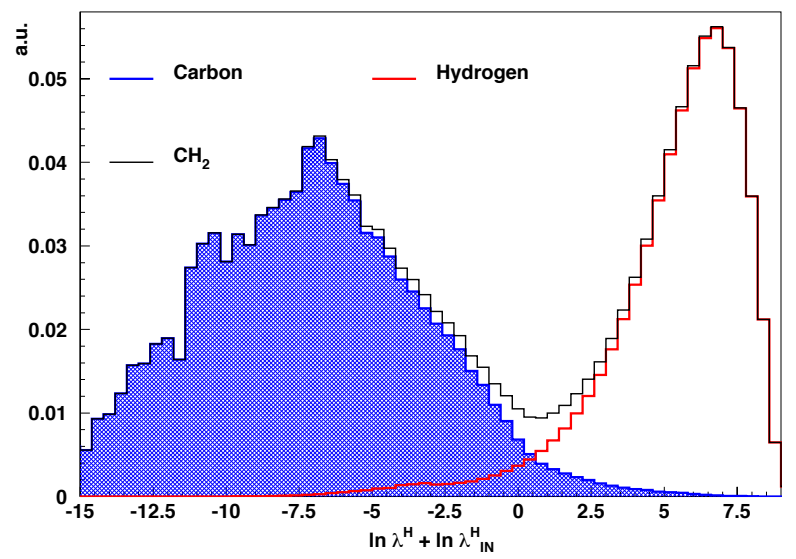

\begin{tabular}{c|c|c} 
Interactions & $\mathrm{CH}_{2}$ & $\mathrm{H}$ \\
\hline \hline \multicolumn{3}{c}{ Standard CP optimized $(1.2 \mathrm{MW}):$} \\
$v_{\mu}$ CC (FHC, 5 y) & $35 \times 10^{6}$ & $3.6 \times 10^{6}$ \\
$\bar{v}_{\mu}$ CC (RHC, 5 y) & $13 \times 10^{6}$ & $2.9 \times 10^{6}$ \\
\hline \multicolumn{3}{c}{ Optimized $v_{\tau}$ appearance $(2.4 \mathrm{MW}):$} \\
$v_{\mu}$ CC (FHC, 2 y) & $66 \times 10^{6}$ & $6.5 \times 10^{6}$ \\
$\bar{v}_{\mu}$ CC (RHC, 2 y) & $24 \times 10^{6}$ & $4.3 \times 10^{6}$ \\
\hline
\end{tabular}

Figure 2: Left panel: Example of kinematic identification of $v_{\mu} p \rightarrow \mu^{-} p \pi^{+}$interactions on hydrogen using multi-dimensional likelihood functions in STT [13]. Right panel: Expected CC statistics in STT (5 tons of $\mathrm{CH}_{2}$ ) with the various LBNF beam options considered [4].

Straw Tube Tracker (STT), in which the neutrino targets are physically separated from the actual tracking system of negligible mass [5]. To achieve high resolution measurements, the target mass is spread out uniformly throughout the entire tracking volume, by keeping the average density low enough - similar to that of liquid deuterium - to have a detector transparent (size comparable to one radiation length) to final state particles produced in neutrino interactions. This detector concept requires to be inserted into a magnetic field and surrounded by a $4 \pi$ electromagnetic calorimeter (ECAL). Examples of different implementations of the STT technology can be found in the CDR of the DUNE experiment $[6,7]$ and in a recently proposed near detector enhancement for the LongBaseline Neutrino Facility (LBNF) [4].

Figure 1 shows a new STT design offering a compact detector equipped with fully configurable neutrino targets, as well as optimized tracking and particle identification. The base tracking technology is provided by low-mass straws similar to the ones used in many modern experiments for precision physics or the search for rare processes $[8,9,10,11,12]$. Thin layers - typically $1-2 \%$ of radiation length - of various target materials (100\% chemical purity) are alternated with straw layers so that they represent about $97 \%$ of the total detector mass (the mass of the straws being $3 \%$ ). This feature, combined with the excellent vertex, angular, momentum, and timing resolutions are key factors to correctly associate neutrino interactions to each target material, as well as for an accurate measurement of the four-momenta of the final state particles. The main target material is $\mathrm{CH}_{2}$ plastic (polypropylene) split between a tunable solid target slab and a radiator (150 foils 15 $\mu m$ thick) for electron identification via transition radiation (Fig. 1). Both the target slab and the radiator can be easily unmounted during data taking, leaving a pure tracking module with average density of $\sim 0.005 \mathrm{~g} / \mathrm{cm}^{3}$. The average density (and fiducial mass) of the detector can be fine tuned up to a maximal value of about $\sim 0.18 \mathrm{~g} / \mathrm{cm}^{3}$. The system composed of the target slab and the radiator can also be replaced by a variety of thin $\left(\ll 0.1 X_{0}\right)$ nuclear targets like $\mathrm{C}, \mathrm{Ca}, \mathrm{Fe}, \mathrm{Pb}, \mathrm{Ar}$, etc., which are interleaved with $\mathrm{CH}_{2}$ modules to guarantee the same acceptance for all targets.

\subsection{Control of Nuclear Effects}

The accurate control of the configuration, chemical composition and mass of the (anti)neutrino targets provided by the STT allows the implementation of a solid hydrogen target by subtracting 


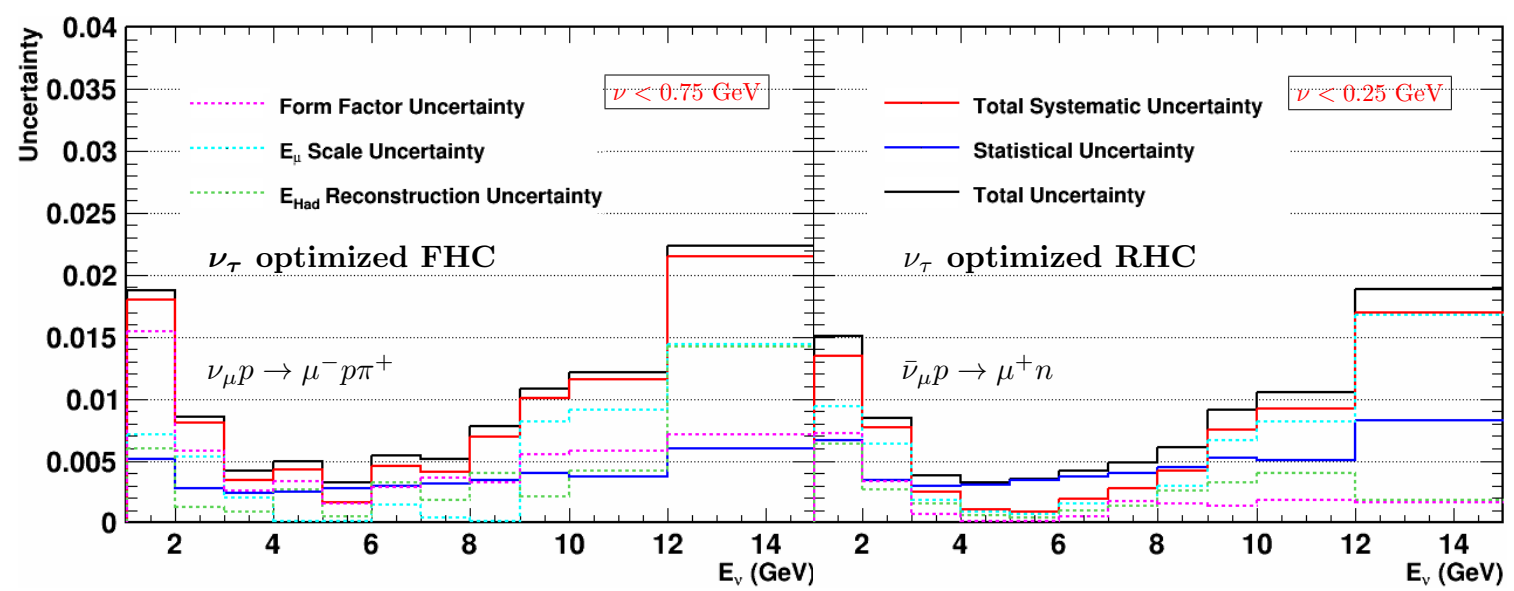

Figure 3: Accuracy in the determination of the $v_{\mu}$ (left panel) and $\bar{v}_{\mu}$ (right panel) relative fluxes using $v_{\mu} p \rightarrow \mu^{-} p \pi^{+}$and $\bar{v}_{\mu} p \rightarrow \mu^{+} n$ exclusive processes on $\mathrm{H}$ with small energy transfer $v$ in STT [14].

measurements on dedicated graphite (pure C) targets from those on the $\mathrm{CH}_{2}$ plastic targets described above [13]. The high resolution of STT allows the identification of the interactions on hydrogen within the $\mathrm{CH}_{2}$ target before subtraction by using a kinematic analysis. Since the $\mathrm{H}$ target is at rest, the Charged Current (CC) events are expected to be perfectly balanced in a plane transverse to the beam direction (up to the tiny beam divergence) and the muon and hadron vectors are back-to-back in the same plane. Instead, events from nuclear targets are affected by both initial and final state nuclear effects, resulting in a significant missing transverse momentum and a smearing of the transverse plane kinematics. By exploit these differences using the reconstructed event kinematics we can achieve efficiencies exceeding $90 \%$ and purities of $80-95 \%$ (Fig. 2) [13]. The subtraction of the small residual background is entirely data-driven by using the corresponding graphite target measurements, which automatically include all types of interactions, as well as reconstruction effects, relevant for the hydrogen selection.

The availability of a solid hydrogen target provides high statistics $v(\bar{v})$-H CC samples (Fig. 2) free from nuclear effects, to be compared with the corresponding ones obtained from the nuclear targets installed within the same detector (same acceptance). This comparison can be performed with both inclusive $\mathrm{CC}$ events and with exclusive topologies and allows a direct measurement of nuclear effects from initial and final state interactions, constraining the corresponding nuclear smearing on the detected interactions. As a result, we can reduce the uncertainties in the unfolding of data collected from heavy targets and precisely calibrate the reconstructed neutrino energy scale.

\subsection{Control of Fluxes}

The solid hydrogen target allows the determination of $v_{\mu}$ and $\bar{v}_{\mu}$ relative fluxes with an accuracy better than $1 \%$ in conventional wide-band neutrino beams, using exclusive $v_{\mu} p \rightarrow \mu^{-} p \pi^{+}$, $\bar{v}_{\mu} p \rightarrow \mu^{+} p \pi^{-}$, and $\bar{v}_{\mu} p \rightarrow \mu^{+} n$ processes on hydrogen with small energy transfer $v$ [14]. This level of precision cannot be achieved with other known techniques using nuclear targets. The hydrogen target solves the problems arising from the nuclear smearing in conventional targets, while the small energy transfer reduces the systematic uncertainties on the energy dependence of the cross-sections. All relevant systematic uncertainties affecting the flux measurements can be directly constrained using data themselves. Figure 3 shows the achievable precisions on the $v_{\mu}$ and $\bar{v}_{\mu}$ relative fluxes with the high-energy LBNF beam option. The measurement of the $\bar{v}_{\mu} p \rightarrow \mu^{+} n$ 
interactions on $\mathrm{H}$ at small momentum transfer $Q$ also provides the absolute $\bar{v}_{\mu}$ flux, since the corresponding cross-section in the limit $Q \rightarrow 0$ is a constant known to high accuracy from neutron $\beta$ decay [14]. The absolute $v_{\mu}$ flux can be accurately determined with the $v e^{-} \rightarrow v e^{-}$elastic scattering, by exploiting the excellent electron identification and angular resolution of STT.

\section{Precision Measurements and Searches}

We discussed a possible way to address the main limitations of neutrino scattering experiments - statistics and resolution, control of targets, nuclear effects, and fluxes - largely reducing the precision gap with respect to electron scattering experiments. These improvements would allow us to exploit the unique properties of the (anti)neutrino probe for precision studies of fundamental interactions and of the structure of nucleons and nuclei $[3,4]$. The near site of the LBNF could then be turned into a general purpose $v \& \bar{v}$ physics facility with a broad program of physics measurements complementary to the ongoing efforts in the fixed-target [15], collider [16], and nuclear physics communities. The level of precision enabled by the techniques described above can provide insights on various fields, unveiling some discovery potential and generating hundreds of diverse physics studies. The same features can also help to reduce the systematic uncertainties affecting the long-baseline neutrino oscillations measurements.

Precision tests of electroweak physics can be performed using complementary channels including the ratio of neutral to charged current $(\mathrm{NC} / \mathrm{CC})$ interactions in $v-\mathrm{N}$ DIS, $v e^{-} \mathrm{NC}$ elastic scattering, ratios of $\mathrm{NC}$ elastic scattering off protons to $\mathrm{CC}$ quasi-elastic scattering, $\mathrm{NC} / \mathrm{CC}$ ratio of coherent $\rho$ production. The unique combination of hydrogen and nuclear targets allows precision studies of the structure of nucleons and nuclei exploiting the flavor selection of the weak current: structure functions and parton distributions, QCD studies, sum rules, high twists and non-perturbative effects, strangeness content of the nucleon, charm production, isospin physics, structure of the weak current, etc. Examples are given by precision tests of the Adler [17] and Gross-Llewellyn Smith [18] sum rules on hydrogen and in nuclei [21, 22]. Similarly, by exploiting the isospin symmetry $F_{2}^{v n}=F_{2}^{\bar{v} p}$ on hydrogen, we can obtain a model-independent measurement of the free neutron structure functions, as well as a measurement of the large $x$ behavior of the $d / u$ quark ratio [19]. These measurements can also be used for precision tests of the isospin (charge) symmetry and would help to elucidate the flavor structure of the nucleon [20]. A complementary program of direct searches for new physics beyond Standard Model can also be performed including a broad range of physics topics like the MiniBooNE anomaly, $v_{\tau}$ appearance and non-standard interactions, Dark Sector physics like heavy sterile neutrinos (e.g. Majorana singlet fermions in $v$ MSM models), axion-like particles, dark photons, light (sub-GeV) dark matter, etc.

\section{References}

[1] L. Alvarez-Ruso et al., "NuSTEC White Paper: Status and challenges of neutrino?nucleus scattering," Prog. Part. Nucl. Phys. 100, 1 (2018) [arXiv:1706.03621 [hep-ph]].

[2] U. Mosel, "Neutrino event generators: foundation, status and future," J. Phys. G 46, no. 11, 113001 (2019) [arXiv:1904.11506 [hep-ex]].

[3] R. Petti, "Precision measurements of fundamental interactions in the Near Detectors," Workshop on Near Detector Physics at Neutrino Experiments, CERN, 18-22 June 2018, https://indico.cern.ch/event/721473/contributions/3034869. 
[4] P. Bernardini et al., "Enhancing the LBNF/DUNE Physics Program," European Particle Physics Strategy Update 2018-2020, https://indico.cern.ch/event/765096/contributions/3295805/.

[5] R. Petti, "NOMAD tracking for T2K," T2K-ND280 Workshop, Rome, 6-8 December 2004, https://www.roma1.infn.it/ ludovici/meeting/talk.html.

[6] S. R. Mishra, R. Petti and C. Rosenfeld, "A High Resolution Neutrino Experiment in a Magnetic Field for Project-X at Fermilab,” PoS NUFACT 08, 069 (2008) [arXiv:0812.4527 [hep-ex]].

[7] R. Acciarri et al. [DUNE Collaboration], "Long-Baseline Neutrino Facility (LBNF) and Deep Underground Neutrino Experiment (DUNE) : Conceptual Design Report, Volume 4 The DUNE Detectors at LBNF," arXiv:1601.02984 [physics.ins-det].

[8] A. Sergi, "NA62 Spectrometer: A Low Mass Straw Tracker,” Phys. Procedia 37, 530 (2012).

[9] M. Anelli et al. [SHiP Collaboration], "A facility to Search for Hidden Particles (SHiP) at the CERN SPS," arXiv:1504.04956 [physics.ins-det].

[10] H. Nishiguchi et al., "Development of an extremely thin-wall straw tracker operational in vacuum ? The COMET straw tracker system,” Nucl. Instrum. Meth. A 845, 269 (2017).

[11] M. Lee, "The Straw-tube Tracker for the Mu2e Experiment," Nuclear and Particle Physics Proceedings 273-275, 2530 (2016), 37th International Conference on High Energy Physics (ICHEP).

[12] P. Gianotti et al., "The Straw Tube Trackers of the PANDA Experiment," arXiv:1307.4537 [physics.ins-det].

[13] H. Duyang, B. Guo, S. R. Mishra and R. Petti, "A Novel Approach to Neutrino-Hydrogen Measurements," arXiv:1809.08752 [hep-ph].

[14] H. Duyang, B. Guo, S. R. Mishra and R. Petti, "A Precise Determination of (Anti)neutrino Fluxes with (Anti)neutrino-Hydrogen Interactions,” Phys. Lett. B 795, 424 (2019) [arXiv:1902.09480 [hep-ph]].

[15] J. Dudek et al., "Physics Opportunities with the 12 GeV Upgrade at Jefferson Lab," Eur. Phys. J. A 48, 187 (2012) [arXiv:1208.1244 [hep-ex]].

[16] A. Accardi et al., "Electron Ion Collider: The Next QCD Frontier : Understanding the glue that binds us all,” Eur. Phys. J. A 52, no. 9, 268 (2016) [arXiv:1212.1701 [nucl-ex]].

[17] S. L. Adler, "Tests of the Conserved Vector Current and Partially Conserved Axial-Vector Current Hypotheses in High-Energy Neutrino Reactions," Phys. Rev. 135, B963 (1964).

[18] D. J. Gross and C. H. Llewellyn Smith, "High-energy neutrino - nucleon scattering, current algebra and partons," Nucl. Phys. B 14, 337 (1969).

[19] S. I. Alekhin, S. A. Kulagin and R. Petti, "Nuclear Effects in the Deuteron and Constraints on the d/u Ratio,” Phys. Rev. D 96, no. 5, 054005 (2017) [arXiv:1704.00204 [nucl-th]].

[20] S. Alekhin, J. Blumlein, S. Kulagin, S. O. Moch and R. Petti, "Strange and non-strange distributions from the collider data,” PoS DIS 2018, 008 (2018) [arXiv:1808.06871 [hep-ph]].

[21] S. A. Kulagin and R. Petti, "Neutrino inelastic scattering off nuclei," Phys. Rev. D 76, 094023 (2007) [hep-ph/0703033 [HEP-PH]].

[22] S. A. Kulagin and R. Petti, "Global study of nuclear structure functions," Nucl. Phys. A 765, 126 (2006) [hep-ph/0412425]. 